\title{
Controlled dephasing of electrons by non-gaussian shot noise
}

\author{
IZHAR NEDER ${ }^{1 *}$, FLORIAN MARQUARDT², MOTY HEIBLUM1ํ, DIANA MAHALU1 AND VLADIMIR UMANSKY1 \\ ${ }^{1}$ Braun Center for Submicron Research, Department of Condensed Matter Physics, Weizmann Institute of Science, Rehovot 76100, Israel \\ ${ }^{2}$ Physics Department, Arnold Sommerfeld Center for Theoretical Physics, and Center for NanoScience, Ludwig-Maximilians-Universität München, \\ 80333 München, Germany \\ *e-mail: izhar.neder@weizmann.ac.il
}

In a 'controlled dephasing' experiment ${ }^{1-3}$, an interferometer loses its coherence owing to entanglement of the interfering electron with a controlled quantum system, which effectively is equivalent to path detection. In previous experiments, only partial dephasing was achieved owing to weak interactions between many detector electrons and the interfering electron, leading to a gaussian-phase randomizing process ${ }^{4-11}$. Here, we report the opposite extreme, where interference is completely destroyed by a few (that is, one to three) detector electrons, each of which has a strong randomizing effect on the phase. We observe quenching of the interference pattern in a periodic, lobe-type fashion as the detector current is varied, and with a peculiar V-shaped dependence on the detector's partitioning. We ascribe these features to the non-gaussian nature of the noise, which is also important for qubit decoherence ${ }^{12}$. In other words, the interference seems to be highly sensitive to the full counting statistics of the detector's shot noise ${ }^{13-16}$.

Our system is based on the previously developed electronic twopath Mach-Zehnder interferometer (MZI $)^{17-20}$. Here, we use two edge channels in the integer quantum Hall effect regime, at filling factor $\mathrm{ff}=2$ (see Fig. 1). The inner edge channel was partitioned and served as a which-path detector. Other than that, the device and the measurement technique were similar to those described in refs 17-19. The MZI was fabricated within a high-mobility twodimensional electron gas. The two paths were formed by splitting the outer edge channel with a quantum-point-contact constriction QPC1. After enclosing a magnetic flux, the two paths joined in QPC2 and interfered. Metallic ohmic contacts served as sources S1, S2 and S3 and drains D1 and D2. Changing the enclosed flux by $\Delta \Phi$ (via the modulation gate, $\mathrm{MG}$ ) changed the Aharonov-Bohm phase $\varphi=2 \pi \Delta \Phi / \Phi_{0}\left(\Phi_{0}=h / e \text { the flux quantum }\right)^{21}$, leading to phase-dependent transmission coefficients; for example, from S2 to D2:

$$
T_{\mathrm{S} 2-\mathrm{D} 2} \equiv T_{\mathrm{MZI}}=\left|t_{\mathrm{QPC} 1} t_{\mathrm{QPC} 2}+\mathrm{e}^{i \varphi} r_{\mathrm{QPC} 1} r_{\mathrm{QPC} 2}\right|^{2}=T_{0}+T_{\varphi} \cos \varphi,
$$

with $t$ and $r$ being the corresponding transmission and reflection amplitudes. The measured visibility, defined as $v=T_{\varphi} / T_{0}$, ranged from $30 \%$ to $60 \%$ (refs $17-19$ ). We attribute the non-ideal visibility to phase fluctuations owing to external noise ${ }^{10}$.

The inner edge channel served as a path detector (see Fig. 1, caption). When QPC0 was tuned to partition the detector channel (which was biased, $V_{\mathrm{det}}=V_{\mathrm{S} 3}$ ), electrons in the upper path of the interferometer became entangled with those in the detector, resulting in a lower visibility. This dephasing process can be considered as 'path detection's or, alternatively, as phase scrambling owing to potential fluctuations in the partitioned detector channel ${ }^{22}$. The interaction between the inner and the outer channels was characterized before the actual dephasing experiment by first fully transmitting and then fully reflecting the biased inner edge channel emanating from S3 (with QPC0). Full transmission $\left(T_{\mathrm{OPCO}}=1\right)$ did not lead to an observable effect on the Aharonov-Bohm oscillations of the MZI as a function of $V_{\mathrm{S} 3}$ (Fig. 2a). However, full reflection $\left(R_{\mathrm{QPC} 0}=1\right)$ had a strong effect on the phase of the interference pattern, which varied linearly with $V_{\mathrm{S} 3}$ (reaching $\sim 2 \pi$ for $\left.V_{\text {det }} \sim 19 \mu \mathrm{V}\right)$, but with nearly no effect on the visibility (Fig. 2b). From this, we can estimate that a mere $n=1-3$ electrons suffice to quench the interference for $V_{\text {det }} \sim 19 \mu \mathrm{V}$ and an interferometer dwell time $\tau=L / v_{\mathrm{g}}$ (with $L \approx 10 \mu \mathrm{m}$ and $\left.v_{\mathrm{g}}=(3-10) \times 10^{6} \mathrm{~cm} \mathrm{~s}^{-1}\right)$. This strong coupling between the edges sets the present experiment apart from previous ones. Note that total dephasing using an adjacent edge channel was also achieved in a quantum $\operatorname{dot}^{23}$.

When QPC0 was tuned to partition the inner channel $\left(0<T_{\mathrm{QPC} 0}<1\right)$, the visibility diminished as $V_{\text {det }}$ increased. Figure 3 shows the dependence of the visibility on $T_{\mathrm{QPC} 0}$ (partitioning) for three different detector voltages. As the bias $V_{\mathrm{S} 3}$ increased, the visibility turned from a smooth parabolic curve to a sharp, $\mathrm{V}$-shaped dependence, with a minimum at $T_{\mathrm{OPC} 0} \sim 0.5$. The dispersion among the experimental points at higher bias resulted from resonances in $T_{\mathrm{QPC} 0}$ (see inset). We argue below that the $\mathrm{V}$-shaped dependence is a signature of the non-gaussian nature of the detector noise.

We first study a simple model where exactly one electron in the detector scrambles the phase of an interfering electron. Detector electrons were injected with a probability $R_{\mathrm{QPC} 0}=1-T_{\mathrm{QPC} 0}$ into the channel that interacted with the interferometer. Depending on the presence or absence of a detector electron, the extra phase, $\delta \varphi$, acquired by an interfering electron fluctuated between two values: $\delta \varphi=\gamma V_{\text {det }}\left(\gamma=(2 \pi / 19) \operatorname{rad} \mu V^{-1}\right.$, from Fig. $\left.2 \mathrm{~b}\right)$ and $\delta \varphi=0$, respectively. Averaging the $\cos \varphi$ term in equation (1) over the two possibilities leads to a visibility ${ }^{2,14}$ :

$$
v=\left|\left\langle\mathrm{e}^{i \delta \varphi}\right\rangle\right|=\left|T_{\mathrm{QPC} 0}+R_{\mathrm{QPC} 0} \mathrm{e}^{i \gamma V_{\mathrm{det}}}\right| .
$$

Equation (2) does not have fitting parameters, so it can be compared directly with the experimental results. Moreover, for 


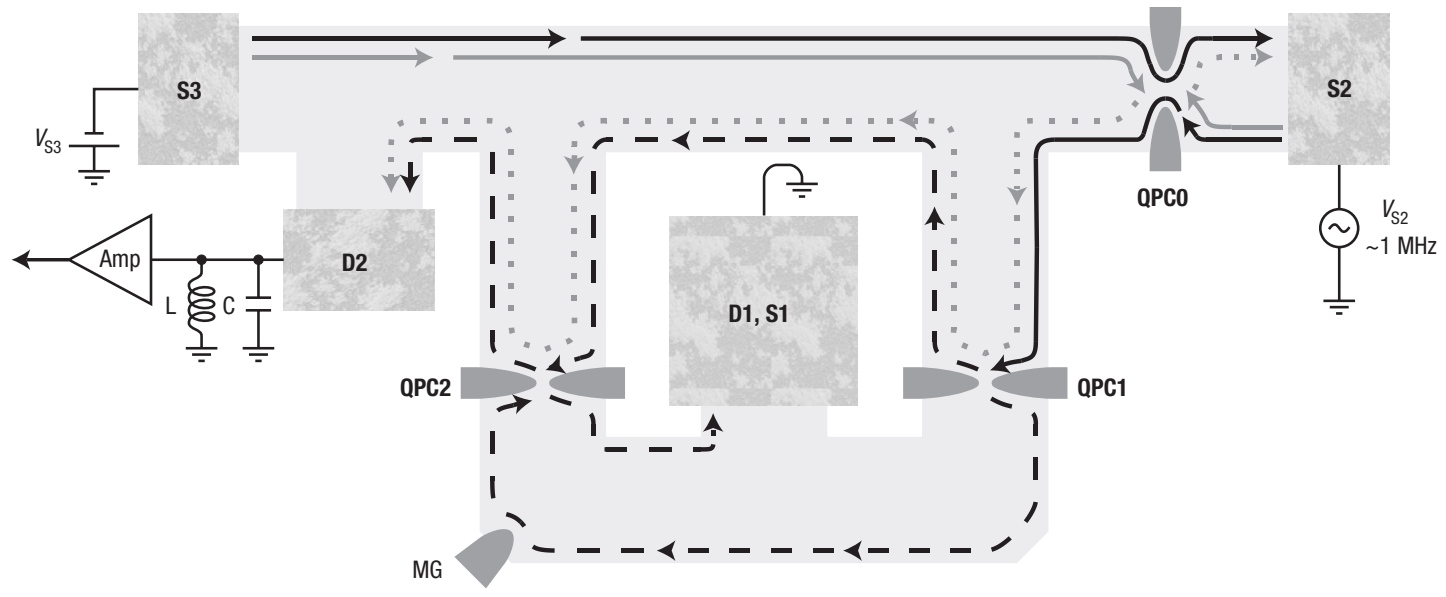

Figure 1 Schematic diagram of the Mach-Zehnder interferometer and the detector. (A scanning electron micrograph of the fabricated structure is given in ref. 19.) The two channels, injected from each source, S2 and S3, propagated towards QPC0, which was tuned to fully transmit the outer channel but partly transmit the inner one. Consequently, two channels impinged on QPC1 from the right: a full outer (interferometer) channel (from S2) and a partitioned inner 'detector' channel (from S2 and S3). QPC1 and QPC2 fully reflected the inner channel and partly transmitted the outer channel (generally $T=R=0.5$ ). The presence of an electron in the upper path of the interferometer affected the phase of the electrons in the detector channel, and vice versa, by Coulomb repulsion.

small $\gamma V_{\text {det }}$ the right-hand side of equation (2) can be expanded to second order:

$$
\begin{aligned}
v & \approx 1-\frac{1}{2}\left(\gamma V_{\mathrm{det}}\right)^{2} T_{\mathrm{QPC} 0}\left(1-T_{\mathrm{QPC} 0}\right) \\
& \approx \exp \left\{-\frac{1}{2}\left(\gamma V_{\mathrm{det}}\right)^{2} T_{\mathrm{QPC} 0}\left(1-T_{\mathrm{QPC} 0}\right)\right\} .
\end{aligned}
$$

This is the so-called gaussian approximation, which depends exponentially only on the second moment of the shot noise, $T_{\mathrm{QPC} 0}\left(1-T_{\mathrm{QPC} 0}\right)$.

The two dotted lines in Fig. $3 \mathrm{a}$ are best fits of a gaussian model to the data, $v \propto \mathrm{e}^{-\alpha T_{\mathrm{QPC}}\left(1-T_{\mathrm{QPC} 0}\right)}$, at detector bias $V_{\text {det }}=4.5 \mu \mathrm{V}$ and $9.5 \mu \mathrm{V}$ (the fitting parameter, $\alpha$, was changed at each bias). For small bias, the induced phase is small $\left(\gamma V_{\text {det }}<\pi / 2\right)$ and both equation (2) and the gaussian approximation with $\alpha=(1 / 2)\left(\gamma V_{\text {det }}\right)^{2}$ agree well with the experimental data seen in Fig. 3a. However, for larger biases, the shape predicted by equation (2) deviates markedly from the smooth gaussian approximation, and has a V-shaped dependence: $v=\left|1-2 T_{\mathrm{QPCO}}\right|$ for $\gamma V_{\mathrm{det}}=\pi\left(V_{\mathrm{det}}=9.5 \mu \mathrm{V}\right)$. Although at this voltage the actual measured data can still be fitted reasonably well by the gaussian model, the fits fail (for any $\alpha$ ) at the even higher detector voltage of $V_{\text {det }}=15 \mu \mathrm{V}$ (shown in Fig. 3b). The V-shaped behaviour is now more obvious (occurring at a higher voltage than predicted by equation (2)).

Another prediction of equation (2) is an oscillatory dependence of the visibility on bias (the coherence should be completely recovered at $\left.\gamma V_{\text {det }}=2 \pi n\right)$. Figure $4 \mathrm{a}$ shows the dependence of the measured visibility and the average phase shift on detector bias (at $T_{\mathrm{QPCO}} \sim 0.5$ ). Whereas in Fig. 3 of ref. 19 the visibility was found to decay monotonously with $V_{\text {det }}$, here we found, in a region of QPC0 gate voltages that was relatively smooth and free of resonances, a non-monotonous decay. The visibility dropped to zero at $V_{\text {det }}=15 \mu \mathrm{V}$ (instead of at $9.5 \mu \mathrm{V}$ according to equation (2)), increased afterwards to reach another, yet smaller, maximum at $V_{\text {det }}=22 \mu \mathrm{V}$, and finally vanished at a higher bias. Moreover, the phase of the Aharonov-Bohm oscillations increased monotonously with $V_{\text {det }}$ (see Fig. $4 \mathrm{~b}$ ): $\langle\delta \varphi\rangle=R_{\mathrm{QPC} 0} \gamma V_{\text {det }}$, but a

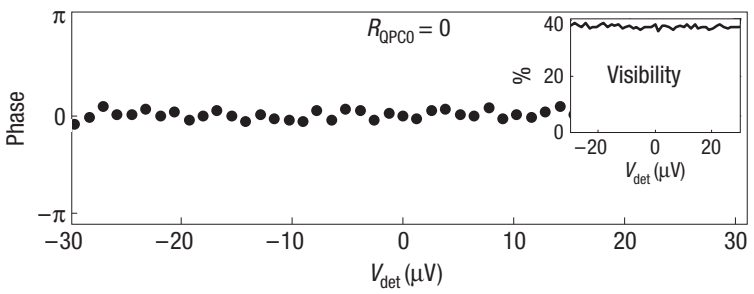

b

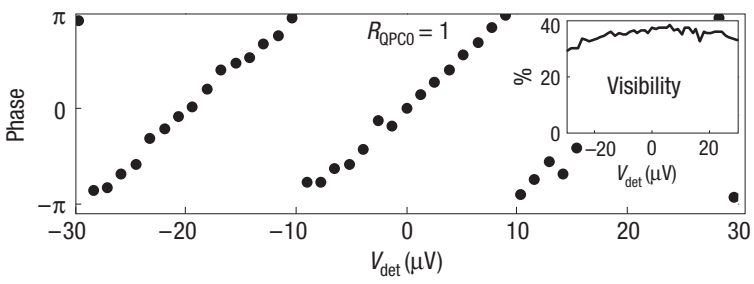

Figure 2 The effect of the inner edge channel on Aharonov-Bohm oscillations in the outer edge channel. $\mathbf{a}$, When the inner ('detector') channel-injected by S3 - is fully transmitted by QPCO $\left(T_{\mathrm{QPCO}}=1\right)$, the phase and visibility at the outer (interferometer) channel are not affected by biasing S3. $\mathbf{b}$, When the inner channel is fully reflected by $\mathrm{QPCO}\left(R_{\mathrm{QPCO}}=1, T_{\mathrm{QPCO}}=0\right)$ and flows parallel and in close proximity to the outer channel upper path, the phase is highly sensitive to the applied bias $V_{\text {det }}$ on $\mathrm{S} 3$, shifting smoothly with $\mathrm{d} \varphi / \mathrm{d} V_{\text {det }} \cong 2 \pi / 19 \mu \mathrm{V}$ (plotted here modulo $2 \pi$ ), whereas the visibility remains almost constant.

underwent a $\pi$-phase slip when the visibility reached zero, as expected qualitatively from equation (2).

The lobe pattern of the quenched visibility observed here resembles the lobe-type evolution of the visibility in a self-biased (by $V_{\mathrm{S} 2}$ ), single-channel $\mathrm{MZI}^{18}$. This similarity suggests that intrachannel interactions (that couple an individual interfering electron to the shot noise produced by the other electrons in the same edge channel) play the role of inter-channel interactions here. Other theoretical work ${ }^{24}$ also finds visibility oscillations in a closely related model. 

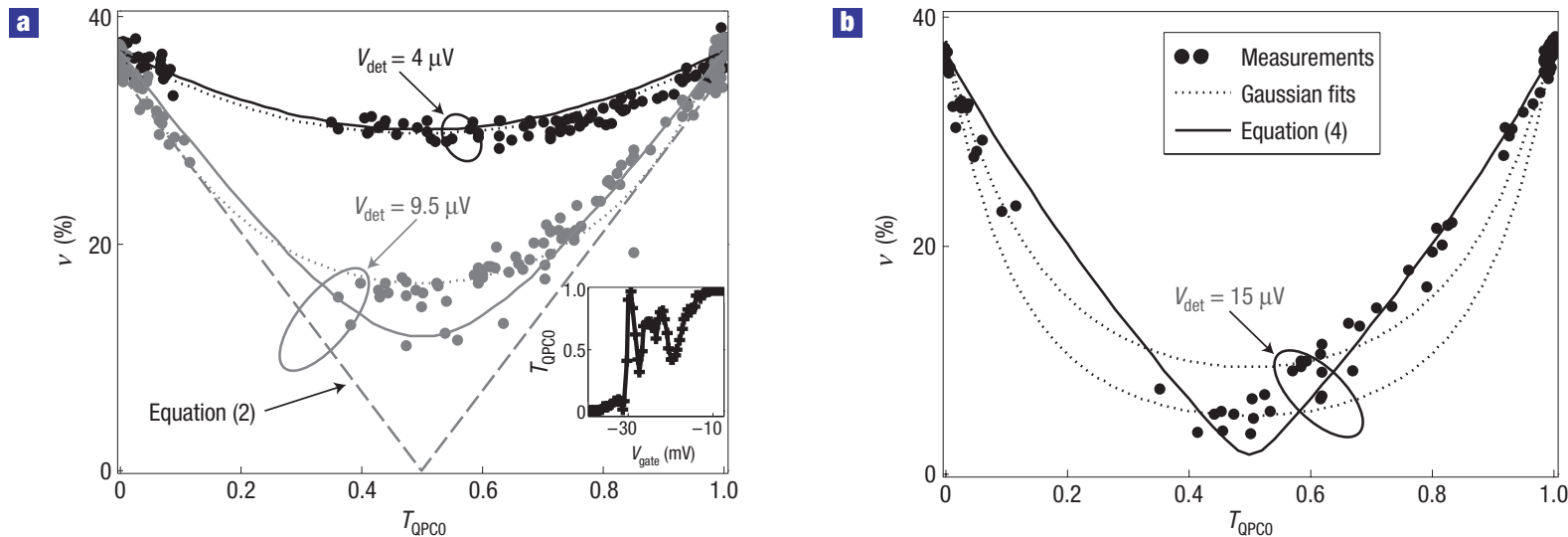

Figure 3 The effect of partitioning the detector channel (by QPCO) on the visibility of the interfering signal, at three different detector bias values. Dotted lines show the best fitted gaussian model; solid lines show the 'microscopic' non-gaussian predictions. As $V_{\text {det }}$ increases, the dependence of the visibility on $T_{\text {opco }}$ turns from a smooth one to a sharp V-shape (at $V_{\text {det }}=15 \mu \mathrm{V}$ ). a, Data points and theoretical predictions for $V_{\text {det }}=4 \mu \mathrm{V}$ and $9.5 \mu \mathrm{V}$. The gaussian model (dotted lines) is adequate at low bias $(4 \mu \mathrm{V})$ and gives a reasonable fit at intermediate bias $(9.5 \mu \mathrm{V})$. The dashed line is the $\mathrm{V}$-shaped prediction of a single-detector-electron model (equation $(2))$ for $V_{\text {det }}=9.5 \mu \mathrm{V}$. Inset: The conductance of QPCO as a function of gate voltage shows sharp resonances. This explains the lack of visibility measurements in the range $0.1<T_{\mathrm{QPCO} 0}<0.4$ and its dispersion at large detector bias (owing to the dependence of the resonances on bias). $\mathbf{b}$, Data points and theoretical predictions for $V_{\text {det }}=15 \mu \mathrm{V}$ (data from ref. 19 ). The $V$ shape is observed experimentally, as predicted by the 'microscopic' non-gaussian model. The gaussian model fails for the arbitrary fitting parameter; the best fit (upper curve) strongly overestimates the visibility at $T_{\mathrm{QPCO}}=0.5$ and underestimates the visibility for $0.7<T_{\mathrm{QPCO}}<0.9$.

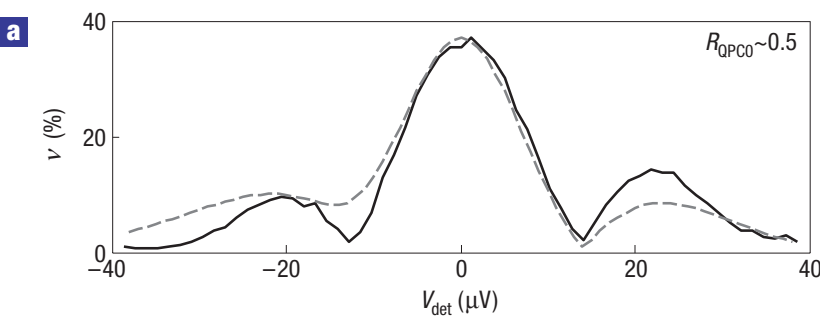

b

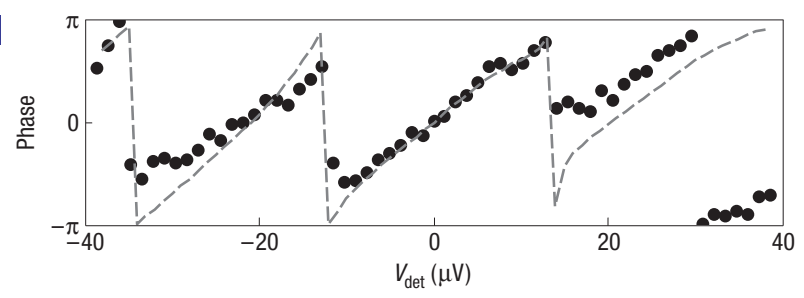

Figure 4 The evolution of the interference pattern as a function of detector voltage, $\boldsymbol{V}_{\text {det }}$, for a partitioned detector channel. a, Visibility evolution. b, Phase evolution (modulo $2 \pi$ ). The non-monotonic behaviour of the visibility is a clear sign of dephasing by non-gaussian noise (data: solid black line and filled circles). Dashed lines: Prediction of the improved theoretical model, which fully accounts for the effects of binomial shot noise, see text, equation (4). The discrepancy at negative $V_{\text {det }}$ results from slight nonlinearity of QPCO, leading to a non-accurate $R_{\mathrm{QPCO}}$ $\left(T_{\mathrm{QPC} 0} \sim 0.5, V_{\mathrm{QPC} O}=-0.0272 \mathrm{~V}\right.$; see inset of Fig. 3a).

To overcome the quantitative shortcomings of equation (2), we now outline a more microscopic approach that predicts the main observed features. A full description of this approach will be provided in a subsequent publication ${ }^{25}$. We assume that every interfering electron accumulates a random phase, $\delta \varphi$, as it traverses the upper arm of the MZI, owing to the coupling with the fluctuating electron density in the detector channel. Treating the detector density, $\rho_{\mathrm{det}}$, classically, the phase should be:

$$
\delta \varphi=\int_{0}^{\tau} \int u\left(v_{\mathrm{g}} t-x\right) \rho_{\mathrm{det}}(x, t) \mathrm{d} x \mathrm{~d} t=\int w(x) \rho_{\mathrm{det}}(x) \mathrm{d} x,
$$

where $u(x)$ is the inter-channel interaction potential, $v_{\mathrm{g}}$ is the electron velocity in the MZI and $\tau$ is the traversal time in the upper path. The electron density, propagating with velocity, $v_{\mathrm{det}}$, obeys: $\rho_{\text {det }}(x, t)=\rho_{\text {det }}\left(x-v_{\text {det }} t, 0\right) \equiv \rho_{\text {det }}\left(x-v_{\text {det }} t\right)$, which yields $w(x)=\int_{0}^{\tau} u\left[\left(v_{\mathrm{g}}-v_{\mathrm{det}}\right) t^{\prime}-x\right] \mathrm{d} t^{\prime}$. It can be shown that equation (3) can be used even in the quantum case $\left(\delta \varphi \mapsto \delta \hat{\varphi}\right.$ and $\left.\rho_{\text {det }} \mapsto \hat{\rho}_{\text {det }}\right)$ to calculate the visibility $v=\left|\left\langle\mathrm{e}^{i \delta \hat{\varphi}}\right\rangle\right|$ as long as the interfering electron is treated in a single-particle picture. This approach neglects Pauli blocking ${ }^{8}$, which has to be taken into account in a phenomenological way when evaluating the visibility. We find (see the Methods section) that the visibility is a product of factors, each in the form of the single-particle expression of equation (2):

$$
v=\left|\left\langle\mathrm{e}^{i \delta \hat{\varphi}}\right\rangle\right|=\prod_{j}\left|T_{\mathrm{QPC} 0}+R_{\mathrm{QPC} 0} \mathrm{e}^{i \delta \varphi_{j}}\right| .
$$

The phases $\delta \varphi_{j}$ are the eigenvalues of the matrix $w_{k^{\prime} k}$ (the Fourier transform of $w(x))$, which has been restricted to transitions between plane-wave states $k^{\prime}, k$ within the voltage window. They depend on the detector voltage, $V_{\text {det }}$, and obey a 'sum rule': $\sum_{j} \delta \varphi_{j}=\gamma V_{\text {det }}$. In the limit $V_{\text {det }} \rightarrow 0$, it can be shown that only one non-zero eigenvalue remains and the result reduces to equation (2) (ref. 25).

Choosing the Fourier transform of $w(x)$ as a lorentzian, with its full-width at half-maximum as the single fitting parameter ( $\Delta=12.4 \mu \mathrm{eV}$; the height being deduced from the observed value of $\gamma$ ), we plotted in Figs 3 and 4 the calculated visibility from equation (4). The plot reproduced the phase slip and zero visibility at $V_{\text {det }}=15 \mu \mathrm{V}$, the second lobe and the eventual decay at higher detector voltages. As more than one detector electron participated 
in the dephasing process, the largest eigenvalue, $\delta \varphi_{1}$, becomes smaller than $\gamma V_{\text {det }}$ (because of the 'sum rule'), such that the zero visibility (when $\delta \varphi_{1}=\pi$ ) is reached at a higher $V_{\text {det }}$ than predicted by equation (2). Although the quality of the fit is good, it may be improved further if $w(x)$, determined by the microscopic physics of the edge channels, were known more precisely. We may conclude that for $V_{\text {det }}<6 \mu \mathrm{V}$ a single detecting electron dephases the MZI, whereas at $V_{\mathrm{det}} \sim 30 \mu \mathrm{V}$ the number is at most three.

In summary, we presented a unique behaviour of an electron interferometer coupled to a which-path detector. Very strong interactions between electrons in both systems led to dephasing by the characteristic binomial, non-gaussian, shot noise in the detector. The dephased visibility had a linear, V-shaped dependence on the partitioning of the detector's current, and non-monotonic, periodic, lobe pattern decay as a function of the detector current. This entanglement between nearly single pairs of electrons may be exploited (in future experiments) to test Bell's inequalities in a system where the detector channel is replaced by another twopath interferometer ${ }^{26-28}$.

\section{METHODS}

\section{SAMPLE AND MEASUREMENTS}

The edges of the sample are defined by plasma etching of a GaAs-AlGaAs heterostructure, embedding a high-mobility two-dimensional electron gas, $80 \mathrm{~nm}$ below the surface. Two edge channels are formed by applying a perpendicular magnetic field of $\sim 3 \mathrm{~T}$, leading to a filling factor of 2 in the bulk (electron temperature $\sim 15 \mathrm{mK}$ ). Transmission of the outer channel to D2 is measured by applying $\sim 1 \mu \mathrm{V}$ at $\sim 1 \mathrm{MHz}$ at S2. The signal at D2 (see Fig. 1), filtered by a cold resonant circuit tuned to $1 \mathrm{MHz}$ with a bandwidth of $30 \mathrm{kHz}$, is amplified by a low-noise preamplifier at $4.2 \mathrm{~K}$. Note that the inner, small, ohmic contact $\left(3 \times 3 \mu \mathrm{m}^{2}\right)$ serves as both D1 and S1. The inner contact was grounded using an air bridge, which had no influence on the transport of the two edge channels underneath.

\section{EVALUATION OF VISIBILITY}

Being a many-particle quantum device, the detector's density cannot be expressed either as a classical function or in a single-particle language. Hence, equation (3) should be rewritten in terms of detector electron operators, $\hat{d}_{k}$, and the matrix elements of $w(x)$ with respect to a plane-wave basis:

$$
\delta \hat{\varphi}=\sum_{k, k^{\prime}} w_{k^{\prime} k} \hat{d}_{k^{\prime}}^{+} \hat{d}_{k}
$$

The occupation of each $k$ state fluctuates independently with $n_{k^{\prime} k} \equiv\left\langle\hat{d}_{k^{\prime}}^{+} \hat{d}_{k}\right\rangle=n_{k} \delta_{k^{\prime} k}$, with $n_{k}=1 / R_{\mathrm{QPC} 0} / 0$ (at zero temperature) for $k$ below/within/above the detector voltage window $E_{\mathrm{F}}<E(k)<E_{\mathrm{F}}+e V_{\text {det }}$. It now becomes possible to express the expectation value of the many-body operator, $\mathrm{e}^{i \delta \hat{\varphi}}$, in terms of a determinant involving the matrices $w_{k^{\prime} k}$ and $n_{k^{\prime} k}$ (ref. 16):

$$
\left\langle\mathrm{e}^{i \delta \hat{\varphi}}\right\rangle=\operatorname{det}\left[1+\left(\mathrm{e}^{i w}-1\right) n\right] .
$$

Equation (5) can be evaluated numerically. It converges in the limit of a large normalization volume and large upper or lower cutoffs in $k$. However, this expression leads to a suppressed visibility even at zero temperature and $V_{\text {det }}=0$, which is an artefact of neglecting the Pauli blocking (which prevents the interfering electrons from scattering into occupied states below $E_{\mathrm{F}}$ ). This can be cured approximately either by rescaling the visibility by a factor independent of $V_{\text {det }}$ (setting it to 1 at $V_{\text {det }}=0$ ), or by restricting the matrix elements $w_{k^{\prime} k}$ to transitions only within the detector voltage window. The latter approach provides a further simplification of equation (5) and yields equation (4) of the main text.

Received 25 October 2006; accepted 23 April 2007; published 3 June 2007.

References

1. Buks, E., Heiblum, M., Mahalu, D. \& Umansky, V. Dephasing in electron interference by a 'which-path' detector. Nature 391, 871-874 (1998).

2. Sprinzak, D., Buks, E., Heiblum, M. \& Shtrikman, H. Controlled dephasing of electrons via a phase sensitive detector. Phys. Rev. Lett. 84, 5820-5823 (2000)

3. Avinun-Kalish, M., Heiblum, M., Silva, A., Mahalu, D. \& Umansky, V. Controlled dephasing of a quantum dot in the Kondo regime. Phys. Rev. Lett. 92, 156801 (2004).

4. Levinson, Y. Dephasing in a quantum dot due to coupling with a quantum point contact. Europhys. Lett. 39, 299-304 (1997)

5. Aleiner, I. L., Wingreen, N. S. \& Meir, Y. Dephasing and the orthogonality catastrophe in tunneling through a quantum dot: The "which path?" interferometer. Phys. Rev. Lett. 79, 3740-3743 (1997).

6. Büttiker, M. \& Martin, A. M. Charge relaxation and dephasing in Coulomb-coupled conductors. Phys. Rev. B 61, 2737-2741 (2000).

7. Levinson, Y. Quantum dot dephasing by edge states. Phys. Rev. B 61, 4748-4753 (1999).

8. Marquardt, F. Fermionic Mach-Zehnder interferometer subject to a quantum bath. Europhys. Lett. 72, 788-794 (2005)

9. Marquardt, F. Equations of motion approach to decoherence and current noise in ballistic interferometers coupled to a quantum bath. Phys. Rev. B 74, 125319 (2006).

10. Marquardt, F. \& Bruder, C. Influence of dephasing on shot noise in an electronic Mach-Zehnder interferometer. Phys. Rev. Lett. 92, 56805 (2004).

11. Seelig, G. \& Büttiker, M. Charge-fluctuation-induced dephasing in a gated mesoscopic interferometer. Phys. Rev. B 64, 245313 (2001).

12. Galperin, Y. M., Altshuler, B. L., Bergli, J. \& Shantsev, D. V. Non-gaussian low-frequency noise as a source of qubit decoherence. Phys. Rev. Lett. 96, 097009 (2006).

13. Levitov, L. S., Lee, H.-W. \& Lesovik, G. B. Electron counting statistics and coherent states of electric current. J. Math. Phys. 37, 4845-4866 (1996).

14. Averin, D. V. \& Sukhorukov, E. V. Counting statistics and detector properties of quantum poin contacts. Phys. Rev. Lett. 95, 126803 (2005).

15. Förster, H., Pilgram, S. \& Büttiker, M. Decoherence and full counting statistics in a Mach-Zehnder interferometer. Phys. Rev. B 72, 075301 (2005).

16. Klich, I. in Quantum Noise in Mesoscopic Physics (ed. Nazarov, Y. V.) 397-402 (NATO Science Series, Kluwer, Dordrecht, 2003).

17. Ji, Y. et al. An electronic Mach-Zehnder interferometer. Nature 422, 415-418 (2003).

18. Neder, I., Heiblum, M., Levinson, Y., Mahalu, D. \& Umansky, V. Unexpected behavior in a two-path electron interferometer. Phys. Rev. Lett. 96, 016804 (2006).

19. Neder, I., Heiblum, M., Mahalu, D. \& Umansky, V. Entanglement, dephasing, and phase recovery via cross-correlation measurements of electrons. Phys. Rev. Lett. 98, 036803 (2007).

20. Litvin, L. V., Tranitz, H.-P., Wegscheider, W. \& Strunk, C. Decoherence and single electron charging in an electronic Mach-Zehnder interferometer. Preprint at <http://arxiv.org/abs/cond-mat/0607758> (2006).

21. Aharonov, Y. \& Bohm, D. Significance of electromagnetic potentials in the quantum theory. Phys. Rev. 115, 485-491 (1959).

22. Stern, A., Aharonov, Y. \& Imry, J. Phase uncertainty and loss of interference: A general picture. Phys. Rev. A 41, 3436-3448 (1990).

23. Rohrlich, D., Zarchin, O., Heiblum, M., Mahalu, D. \& Umansky, V. Controlled dephasing of a quantum dot: From coherent to sequential tunneling. Phys. Rev. Lett. 98, 096803 (2007).

24. Sukhorukov, E. V. \& Cheianov, V. V. Resonant dephasing of the electronic Mach-Zehnder interferometer. Preprint at $<$ http://arxiv.org/abs/cond-mat/0609288 $>$ (2006).

25. Neder, I. \& Marquardt, F. Coherence oscillations in dephasing by non-gaussian shot noise. New J. Phys. 9, 1-30 (2007).

26. Beenakker, C. W. J., Emary, C., Kindermann, M. \& van Velsen, J. L. Proposal for production and detection of entangled electron-hole pairs in a degenerate electron gas. Phys. Rev. Lett. 91 147901 (2003).

27. Samuelson, P., Sukhorukov, E. V. \& Büttiker, M. Two-particle Aharonov-Bohm effect and entanglement in the electronic Hanbury Brown-Twiss setup. Phys. Rev. Lett. 92, 026805 (2004). 28. Bell, J. S. On the Einstein-Podolsky-Rosen paradox. Physics 1, 195-200 (1964).

\section{Acknowledgements}

We are indebted to Y. Levinson for helpful discussions. The work was partly supported by the Israeli Science Foundation (ISF), the Minerva foundation, the German Israeli Foundation (GIF), the SFB 63 of the DFG, the Nanosystems Initiative Munich (NIM) and the German Israeli Project cooperation (DIP).

Correspondence and requests for materials should be addressed to I.N.

\section{Competing financial interests}

The authors declare no competing financial interests.

Reprints and permission information is available online at http://npg.nature.com/reprintsandpermissions/ 